\title{
Imaging and Methotrexate Response Monitoring of Systemic Inflammation in Arthritic Rats Employing the Macrophage PET Tracer $\left[{ }^{18}\right.$ F] Fluoro-PEG-Folate
}

\author{
Durga M. S. H. Chandrupatla $\left(\mathbb{D},{ }^{1}\right.$ Gerrit Jansen, ${ }^{1}$ Elise Mantel, ${ }^{1}$ Philip S. Low, ${ }^{2}$ \\ Takami Matsuyama, ${ }^{3}$ René P. Musters, ${ }^{4}$ Albert D. Windhorst, ${ }^{5}$ Adriaan A. Lammertsma $\mathbb{D}^{5},{ }^{5}$ \\ Carla F. M. Molthoff $\mathbb{D}^{5},{ }^{5}$ and Conny J. van der Laken $\mathbb{D}^{1}$ \\ ${ }^{1}$ Amsterdam Rheumatology and Immunology Center, Location VUmc, VU University Medical Center, Amsterdam, Netherlands \\ ${ }^{2}$ Department of Chemistry, Purdue University, West Lafayette, IN, USA \\ ${ }^{3}$ Department of Immunology, Graduate School of Medical and Dental Sciences, Kagoshima University, Kagoshima 890-8544, Japan \\ ${ }^{4}$ Department of Physiology, VU University Medical Center, Amsterdam, Netherlands \\ ${ }^{5}$ Department of Radiology \& Nuclear Medicine, VU University Medical Center, Amsterdam, Netherlands
}

Correspondence should be addressed to Conny J.van der Laken; j.vanderlaken@vumc.nl

Received 22 September 2017; Revised 22 December 2017; Accepted 24 January 2018; Published 21 February 2018

Academic Editor: Cristina Nanni

Copyright (c) 2018 Durga M. S. H. Chandrupatla et al. This is an open access article distributed under the Creative Commons Attribution License, which permits unrestricted use, distribution, and reproduction in any medium, provided the original work is properly cited.

\begin{abstract}
Background. In rheumatoid arthritis, articular inflammation is a hallmark of disease, while the involvement of extra-articular tissues is less well defined. Here, we examined the feasibility of PET imaging with the macrophage tracer $\left[{ }^{18} \mathrm{~F}\right]$ fluoro-PEG-folate, targeting folate receptor $\beta(\mathrm{FR} \beta)$, to monitor systemic inflammatory disease in liver and spleen of arthritic rats before and after methotrexate (MTX) treatment. Methods. $\left[{ }^{18} \mathrm{~F}\right]$ Fluoro-PEG-folate PET scans $(60 \mathrm{~min})$ were acquired in saline- and MTX-treated $(1 \mathrm{mg} / \mathrm{kg}, 4 \mathrm{x})$ arthritic rats, followed by tissue resection and radiotracer distribution analysis. Liver and spleen tissues were stained for ED1/ED2macrophage markers and FR $\beta$ expression. Results. $\left[{ }^{18} \mathrm{~F}\right]$ Fluoro-PEG-folate PET and ex vivo tissue distribution studies revealed a significant $(p<0.01)$ 2-fold lower tracer uptake in both liver and spleen of MTX-treated arthritic rats. Consistently, ED1- and ED2-positive macrophages were significantly $(p<0.01)$ decreased in liver (4-fold) and spleen (3-fold) of MTX-treated compared with saline-treated rats. Additionally, FR $\beta$-positive macrophages were also significantly reduced in liver $(5$-fold, $p<0.005)$ and spleen (3-fold, $p<0.01$ ) of MTX- versus saline-treated rats. Conclusions. MTX treatment reduced activated macrophages in liver and spleen, as markers for systemic inflammation in these organs. Macrophage PET imaging with $\left[{ }^{18} \mathrm{~F}\right]$ fluoro-PEG-folate holds promise for detection of systemic inflammation in RA as well as therapy (MTX) response monitoring.
\end{abstract}

\section{Introduction}

Rheumatoid arthritis (RA) is a chronic inflammatory disease involving mainly the synovium of the joints, although other tissue/organ involvement has been recognized $[1,2]$. Extra-articular manifestations occur in active and severe RA, including skin, eye, heart, lung, renal, nervous, and gastrointestinal systems $[3,4]$. Therefore, early detection and treatment of systemically affected organs in RA could benefit in achieving predefined low disease activity and remission $[5,6]$. To this end, in a preclinical setting, animal models of arthritis may serve a valuable tool for imaging (extra) articular and nonarticular inflammation and for monitoring the response to therapeutic interventions.

Many experimental animal models have been exploited to unravel the pathophysiology of inflammatory arthritis [712]. However, in most of these studies the primary research focus was on disease pathways and immune cells of the synovium rather than extra-articular manifestations. Also, depending on the modality and time frame of arthritis induction, extra-articular manifestations were not monitored or underreported. 
Macrophages are known to play central role in RA disease progression [13]. Several studies have shown a direct correlation between disease remission and lower numbers of macrophage infiltration incidents into the synovium [14-16]. In patients, tissue resident macrophages in macrophage-rich organs such as liver and spleen may also be involved in extraarticular inflammation in RA [17]. Recent studies indicated that up to $50 \%$ of RA patients were reported with abnormal liver symptoms, including elevated alkaline phosphatase and small foci of necrosis and fatty liver [18]. Moreover, liver resident macrophages in an animal model were implicated in regulating chronic inflammation of arthritis through interacting with synovial phagocytes [19]. Not limiting to liver, spleen has also been reported in systemic inflammation in RA. Studies have shown manifestations of spleen enlargement and histological changes in either early or longstanding RA [20, 21].

Macrophage Positron Emission Tomography (PET) has been proposed as a noninvasive modality to monitor disease activity and therapy response in the whole body [22]. Beyond the prototypical macrophage tracer $\left[{ }^{11} \mathrm{C}\right]-\mathrm{PK} 11195$, targeting the translocator protein (TSPO) on activated macrophages, second-generation TSPO tracers showed improved properties over $\left[{ }^{11} \mathrm{C}\right]-\mathrm{PK} 11195$ to visualize arthritis [23]. Other interesting macrophage PET tracers to visualize arthritis are 4- $\left[{ }^{18} \mathrm{~F}\right]$-fluorophenylfolate, $\left[{ }^{68} \mathrm{Ga}\right]$-DOTA-folate [24], and $\left[{ }^{18} \mathrm{~F}\right]$ fluoro-PEG-folate [25]. These folate-based tracers bind with high affinity to folate receptor $\beta(\mathrm{FR} \beta)$ expressed on activated macrophages [26-28]. FR $\beta$ is also of interest from a therapeutic perspective as it can bind and internalize antifolates and folate-conjugated antiarthritic therapeutics [26-31].

Recently we reported that the macrophage tracer $\left[{ }^{18} \mathrm{~F}\right]$ fluoro-PEG-folate allowed visualizing arthritis in the inflamed knee joints of arthritic rats and also was able to monitor the response to the anchor drug in RA therapy, methotrexate (MTX) [32]. In the present study we extend on these observations by exploiting $\left[{ }^{18} \mathrm{~F}\right]$ fluoro-PEG-folate PET to monitor potential systemic inflammation in liver and spleen of arthritic rats before and after MTX therapy, hypothesizing that MTX therapy also impacts systemic inflammatory effects in the organs. These studies were complemented with histological and immunofluorescence assessment of macrophage infiltration in liver and spleen.

\section{Materials and Methods}

2.1. Animals. The European community council directives 2010/63/EU for laboratory animal care and the Dutch law on animal experimentation criteria were fulfilled for performing the animal experiments. Wister rats (male, 150-200 grams, Charles River International Inc., Sulzfeld, Germany) were provided with standard food, water (ad libitum), and conditions as described previously [32]. The local committee on animal experimentation of the VU University Medical Center (DEC PET13-07) validated and approved experimental protocols.

2.2. Arthritic Induction and Therapeutic Interventions. Wistar rats were immunized [33] and arthritis was induced via
$4 \mathrm{x}$ intra-articular (i.a.) methylated bovine serum albumin (mBSA) injections, 4 or 5 days apart in the arthritic (right) knee with the contralateral (left, nonarthritic) knee serving as control knee essentially as described before [33]. Rats were anesthetized during immunization and arthritic induction using inhalation anesthetics (isoflurane: $2-2.5 \%$ and oxygen: $1 \mathrm{~L} / \mathrm{min})$.

After the last i.a. injection the rats ( $n=4$ /group) were treated $4 \mathrm{x}(\mathrm{d} 0, \mathrm{~d} 7, \mathrm{~d} 14$, and $\mathrm{d} 21)$ either with saline $(500 \mu \mathrm{L}$, intraperitoneal (i.p.) injection) or with MTX (VU University Medical Centers' Pharmacy) (i.p.) at $1.0 \mathrm{mg} / \mathrm{kg}$. Healthy rats (nonarthritic) $(n=3)$ did not receive either arthritic induction or therapeutic interventions [32].

Six days after the last saline or MTX treatment, $\left[{ }^{18} \mathrm{~F}\right]$ fluoro-PEG-folate PET scans were performed, immediately after which rats were sacrificed and tissues were excised for further processing and various analyses described hereafter.

2.3. $\left[{ }^{18}\right.$ F]Fluoro-PEG-Folate and PET. $\left[{ }^{18} \mathrm{~F}\right]$ fluoro-PEG-folate was synthesized as previously described [25], with a radiochemical purity of $>97 \%$ and mean specific activity of $49.7 \pm 2.1 \mathrm{GBq} / \mu \mathrm{mol}$. Saline- and MTX-treated arthritic rats were anesthetized using inhalation anaesthetics (isoflurane: $2-2.5 \%$ and oxygen: $1 \mathrm{~L} / \mathrm{min}$ ). The jugular vein was cannulated with a polyurethane 3 -French cannula $(0.7 \mathrm{~mm}$ $\times 19 \mathrm{~mm}, \mathrm{BD}$ Angiocath, Breda, Netherlands). During all procedures body temperature, heartbeat, respiratory rate, and blood oxygen saturation were monitored continuously using a rectal temperature probe and a pulse oxygen meter with $\mathrm{SpO}_{2}$ sensor. Anesthetized rats $(n=2$, from saline- and MTX-treated groups) were positioned in a high resolution research tomograph (HRRT) (Siemens/CTI, Knoxville, TN, USA) and $\left[{ }^{18} \mathrm{~F}\right]$ fluoro-PEG-folate $(20.5 \pm 3.4 \mathrm{MBq})$ was administered i.v. through the cannula and a dynamic PET scan was acquired for $60 \mathrm{~min}$. Next, PET scans were normalized (for scatter, random, attenuation, decay, and dead time) and reconstructed as described before [25]. AMIDE software (version 0.9.2) [34] was used to analyse the images and data were expressed as standardized uptake values (SUV). The last frame was used to manually draw fixed size ellipsoidal shaped ROI over the area of liver and spleen (dimensions: $4 \times 4 \times$ $4 \mathrm{~mm}^{3}$ ) and arthritic and contralateral knees (dimensions: 7 $\times 4 \times 7 \mathrm{~mm}^{3}$ ). The ROI for knees was drawn on top of the knee area [25] whereas, for liver and spleen, first a dotted line was drawn to represent the organ and then ROI was drawn approximately at the same spot in the saline- and MTXtreated rats. Through projecting ROIs onto the dynamic image sequence the time activity curve (TAC) was generated. TACs were expressed as standardized uptake values (SUV), that is, mean ROI radioactivity concentration normalized to injected dose and body weight.

2.4. Ex Vivo Tissue Distribution Studies. Rats (saline $(n=4)$, $\operatorname{MTX}(n=4))$ were sacrificed sixty minutes after $\left[{ }^{18} \mathrm{~F}\right]$ fluoroPEG-folate tracer administration [33]. Upon sacrificing, the knees, liver, and spleen were excised, rinsed, dipped dry, weighed, and the amount of radioactivity determined using an LKB 1282 Compugamma CS gamma counter (LKB, 
Wallac, Turku, Finland). Tissue radioactivity was expressed as percentage of the injected dose per gram tissue (\%ID/g).

2.5. Histopathology and Immunohistochemistry. The liver and spleen sections from all rats $(n=3$ for healthy rats and $n=4$ for saline- and MTX-treated rats) were fixed in $4 \%$ neutral buffered paraformaldehyde for $24 \mathrm{~h}$ before embedding in paraffin wax. Sections of $5 \mu \mathrm{m}$ were cut and stained initially with haematoxylin and eosin and then with an ED1 (homologous to human CD68), ED2 (homologous to human CD163), or isotype control antibody [32]. ED2/CD163 serves as marker for M2-type (anti-inflammatory) macrophages. Images were captured using a Leica 4000B microscope and Leica digital camera DC500 (Microsystems B.V. Rijswijk, Netherlands).

\subsection{FR $\beta$ Immunofluorescence and Microscopy (Frozen Rat} Tissue). At the end of the study, liver and spleen tissues were collected from healthy rats $(n=3)$ and saline- and MTXtreated rats $(n=4)$ and snap frozen in liquid nitrogen and stored at $-80^{\circ} \mathrm{C}$. Tissues were embedded in appropriate media (OCT; SKU4583, Tissue-Tek, Netherlands) and were cut using cryotome $\left(-20^{\circ} \mathrm{C}\right)$ (Leica, Netherlands) and placed on Superfrost (4951PLUS4, ThermoFisher, Netherlands) glass slides for immunofluorescence (IF) staining. Sections of $8 \mu \mathrm{m}$ were cut and stained with haematoxylin and eosin, and staining for FR $\beta$-positive macrophages was performed with a mouse anti-rat FR $\beta$ antibody [29] or isotype control antibody.

For immunostaining, liver and spleen tissue sections were first brought to room temperature (RT) for $30 \mathrm{~min}$, fixed in acetone (439126, Sigma-Aldrich, Netherlands) for $10 \mathrm{~min}$ at $-20^{\circ} \mathrm{C}$, and air-dried for $10 \mathrm{~min}$ at RT. A DAKO pen was used to mark the sections (S2002, DAKO, Santa Carla, CA, USA) which were subsequently washed $3 \mathrm{x}$ with PBS on a shaker. Next, sections were incubated with $100 \%$ fetal bovine serum (FBS) for $30 \mathrm{~min}$ at RT to avoid nonspecific binding and washed again in PBS $(3 \times 5 \mathrm{~min})$. Thereafter, sections were incubated with mouse anti-rat FR $\beta$ IgM (final concentration $1 \mu \mathrm{g} / \mathrm{ml}$ ) or isotype control IgM (ab35768, Abcam, Cambridge, UK; final concentration $1 \mu \mathrm{g} / \mathrm{ml}$ ) in $10 \%$ FBS/PBS for 24 hours at $4^{\circ} \mathrm{C}$ or with $10 \% \mathrm{FBS} / \mathrm{PBS}$. After washing $(3 \times 5 \mathrm{~min}$ in PBS on a shaker), sections were incubated with goat-anti-mouse Alexa 488 ((final concentration $1 \mu \mathrm{g} / \mathrm{ml}$ ) (A21042) ThermoFisher Scientific, Netherlands) in $10 \% \mathrm{FBS} / \mathrm{PBS}$, washed $(3 \times 5 \mathrm{~min}$ in $\mathrm{PBS}$ on a shaker $)$, air-dried, and mounted with $2 \mu \mathrm{l}$ of MOWIOL mounting medium (81381, Merck, Zwijndrecht, The Netherlands). The 2D IF slides were imaged with a Zeiss Axiovert $200 \mathrm{M}$ Marianas $^{\mathrm{TM}}$ inverted microscope (40x oil-immersion lens). The microscope, camera, and data processing were controlled by SlideBook ${ }^{\mathrm{TM}}$ software (SlideBook version 6 (Intelligent Imaging Innovations, Denver, CO)) as described previously [35].

2.7. Quantification of Macrophages. The identity of all stained slides was hidden from and counted by two independent observers for FR $\beta$ and ED1- and ED2-positive macrophages. For quantification, representative areas of liver and spleen sections were divided into 4 regions and counted at 400x magnification for FR $\beta$ and ED1- and ED2-positive macrophages in the saline- and MTX-treated rats. The average numbers of macrophages per area from all four regions were combined and depicted as total numbers of FR $\beta$, ED1, or ED2 macrophages.

2.8. Statistical Analysis. Statistical analysis was performed using SPSS (version 15) for Windows (SPSS Inc., Chicago, IL, USA). Mann-Whitney (exact) tests were performed to analyse differences in tracer uptake (tissue distribution) macrophage infiltration in saline- versus MTX-treated groups. A $p$ value $<0.05$ was considered as statistically significant. All results are presented as mean \pm standard deviation (SD).

\section{Results and Discussion}

3.1. Arthritis Induction and MTX Therapeutic Interventions. Upon arthritic induction all rats showed macroscopic thickening of the arthritic knee compared with the contralateral control knee (data not shown). As shown earlier, arthritis induction was well tolerated and allowed a window for therapeutic intervention with MTX, which was also well tolerated and not associated with any adverse effects [32].

3.2. $\left[{ }^{18}\right.$ F]Fluoro-PEG-Folate PET. In a recent study we showed that imaging with the macrophage tracer $\left[{ }^{18} \mathrm{~F}\right]$ fluoroPEG-folate could visualize decreased accumulation of the tracer in the knee joints of arthritic rats treated with MTX. In the present study, we particularly focussed on macrophagerich organs such as liver and spleen for their potential involvement in systemic inflammation and the impact of MTX therapy upon this. The coronal PET images visualized higher tracer uptake in liver and spleen of the saline-treated arthritic rats (Figure 1(a)) compared to the MTX-treated rats (Figure 1(b)). Standard uptake values (SUV) of $\left[{ }^{18} \mathrm{~F}\right]$ fluoroPEG-folate were quantified for liver and spleen with ROIs (colored ellipsoid) demonstrating decreased liver (1.5-fold) (Figure 1(c)) and spleen (2-fold) (Figure 1(d)) tracer uptake in MTX-treated compared to saline-treated rats. The relatively high uptake in the intestinal area, kidney, and bladder was due to the known clearance of the folate tracer $[25,32]$. The MTX treatment results showed that, beyond knee joints, folate tracer binding is also inhibited by methotrexate in the extra-articular tissues, liver, and spleen, which suggests local anti-inflammatory effects on macrophage activity as part of systemic inflammation in these organs. These results are consistent with data from another arthritic rat model wherein $\left[99^{\mathrm{m}} \mathrm{Tc}\right]-\mathrm{EC} 20$ folate scans also showed increased tracer uptake in liver and spleen [36] as compared to healthy rats. The increased tracer uptake in liver and spleen in arthritic rats coincided with increased tissue FR levels as measured by $\left[{ }^{3} \mathrm{H}\right]$ folic acid binding studies. Notably, a clinical study with $\left[{ }^{18} \mathrm{~F}\right]-\mathrm{FDG}$, an indicator of active metabolism, in patients with collagen vascular disease-associated arthritis also showed significantly increased tracer uptake in the spleen, pointing to its inflammatory involvement [37]. In a clinical study in RA patients with $\left[99^{\mathrm{m}} \mathrm{Tc}\right]-\mathrm{EC} 20$ folate, articular inflammation as well as liver and spleen involvement were demonstrated [38], further corroborating systemic inflammatory effects in arthritis. 


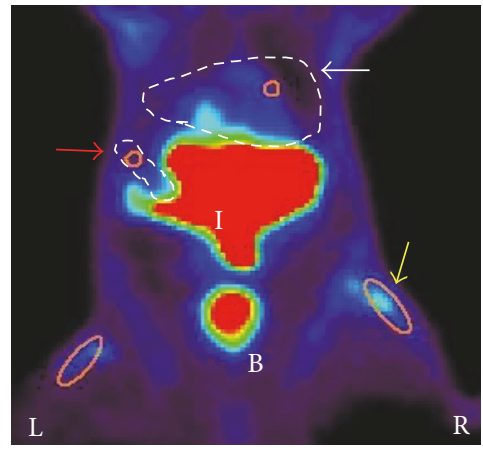

(a)

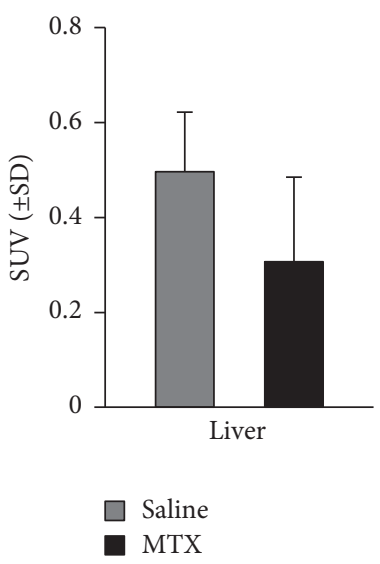

(c)
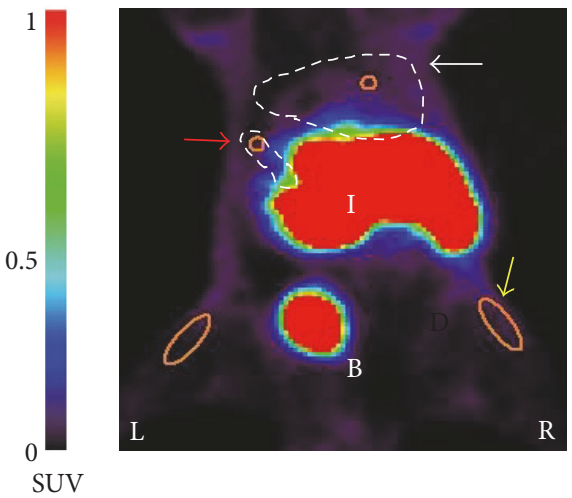

(b)

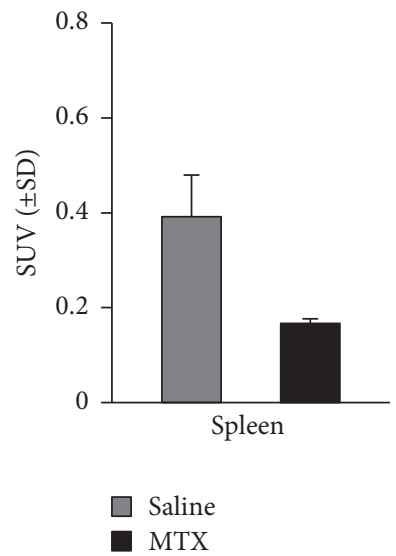

(d)

FIGURE 1: Representative coronal PET images of $\left[{ }^{18} \mathrm{~F}\right]$ fluoro-PEG-folate in (a) saline-treated $(n=2)$ and (b) MTX-treated $(n=2)$ rats. Orange ellipsoid: ROI drawn around the synovium of the knee joint, liver (white arrow), spleen (red arrow), and arthritic (right (R); yellow arrow) and contralateral knees (left $(L))$ depicted on each image. Spleen and liver areas are indicated by dashed lines. Standardized uptake value (SUV) scale bar from minimum 0 to maximum 1 represents the uptake of the tracer. Clearance organs intestine (I) and bladder (B) are also depicted. $\left[{ }^{18}\right.$ F]Fluoro-PEG-folate uptake is expressed as SUV $( \pm S D)$ in $(c)$ liver and $(d)$ spleen of the saline- and MTX-treated group.

3.3. Ex Vivo Tissue Distribution Studies. To further establish the usefulness of therapeutic monitoring of systemic inflammation via $\left[{ }^{18} \mathrm{~F}\right]$ fluoro-PEG-folate PET and regular MTX treatment (the anchor drug in RA), ex vivo tissue distribution studies were performed on selected tissues 60 minutes after tracer injection. In excised liver and spleen sections of MTXtreated rats, tracer uptake was significantly 3 - and 16 -fold lower ( $p<0.01$ and $p<0.001$ ), respectively, compared to the saline-treated rats (Figure 2). For comparison, previously reported tracer uptake in liver and spleen of healthy rats was $\sim 1.4$-fold lower [32] than in arthritic rats also pointing at presence of systemic inflammation/macrophage activity in liver and spleen. The markedly lower tracer uptake (5fold, $p<0.01$ ) in the MTX-treated arthritic rat knees [32] is depicted as a reference (Figure 2). Plasma levels of $\left[{ }^{18} \mathrm{~F}\right]$ fluoro-PEG-folate were low and comparable between both groups. Uptake of $\left[{ }^{18} \mathrm{~F}\right]$ fluoro-PEG-folate in kidney $(2.92 \pm 0.33$ versus $3.34 \pm 0.63 \% \mathrm{ID} / \mathrm{g})$ and intestine $(1.06 \pm 0.49$ versus $0.84 \pm 0 / 56 \% \mathrm{ID} / \mathrm{g}$ ) is not significantly altered after MTX therapy. This is consistent with the notion that kidney constitutively expresses another FR isoform (i.e., $\mathrm{FR} \alpha$, implicated in renal retention of folates) [32].
It is of importance to note that tissue distributions data were obtained 6 days after the last MTX administration; thus it is unlikely that lowered tracer uptake is due to FR $\beta$ blocking by MTX as residual plasma levels of MTX will be very low $(<10 \mathrm{nM})$ at that stage [32]. Moreover, $\left[{ }^{18} \mathrm{~F}\right]$ fluoro-PEG-folate binding affinity towards FR $\beta$ outweighs MTX by at least 2-3 orders of magnitude $[25,27]$.

Together, PET and tissue distribution data illustrate that MTX treatment has a marked effect on macrophage tracer uptake in liver and spleen of arthritic rats.

3.4. Effect of MTX on Systemic Macrophage Infiltration. To extend on the PET and ex vivo tissue distribution data with $\left[{ }^{18} \mathrm{~F}\right]$ fluoro-PEG-folate, the level of macrophage infiltration was examined in saline-treated and MTX-treated rats. Macrophage numbers were quantified in liver and spleen sections of saline-treated versus MTX-treated rats by immunohistochemical assessment of the abundance of total ED1-positive macrophages and ED2-positive macrophages, the latter, as CD163 homologue, serving as a proposed marker for anti-inflammatory macrophages. Figures 3 and 4 show representative images of ED1- and ED2-positive macrophages 


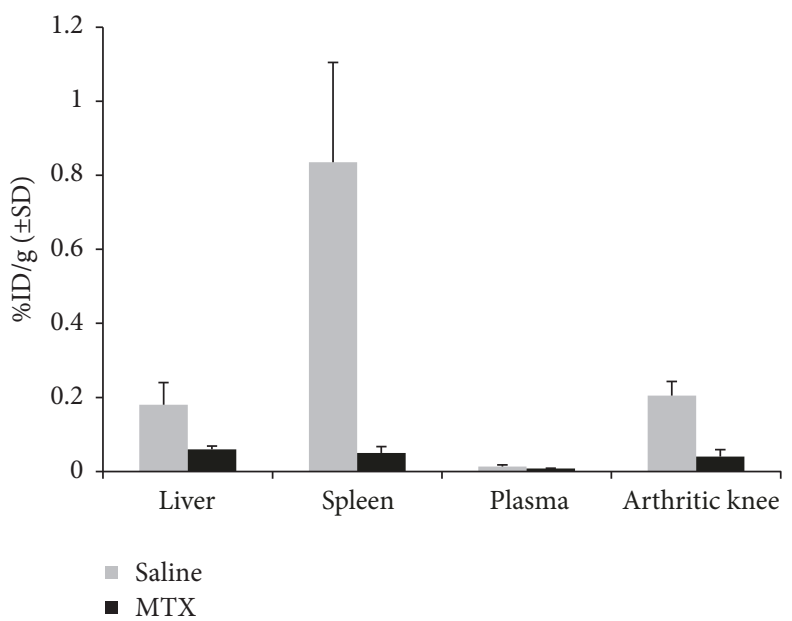

FIGURE 2: Ex vivo tissue distribution of $\left[{ }^{18} \mathrm{~F}\right]$ fluoro-PEG-folate in liver, spleen, plasma, and arthritic knee of saline-treated $(n=4)$ and MTXtreated $(n=4)$ rats at $60 \mathrm{~min}$ after tracer injection. Results expressed as mean percentage injected dose per gram (\%ID/g). Error bars indicate SD. $p<0.01$ and $p<0.001$.

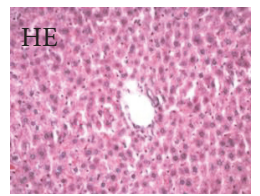

$\mathrm{ED} 1+$

Healthy (ED1)

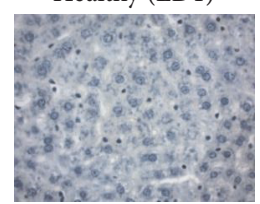

ED1-

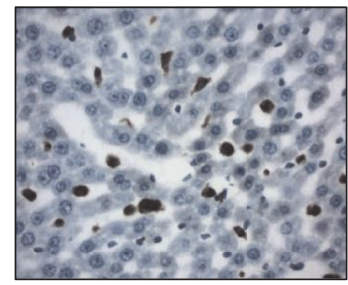

(a)

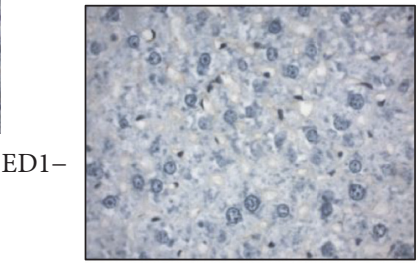

(c)

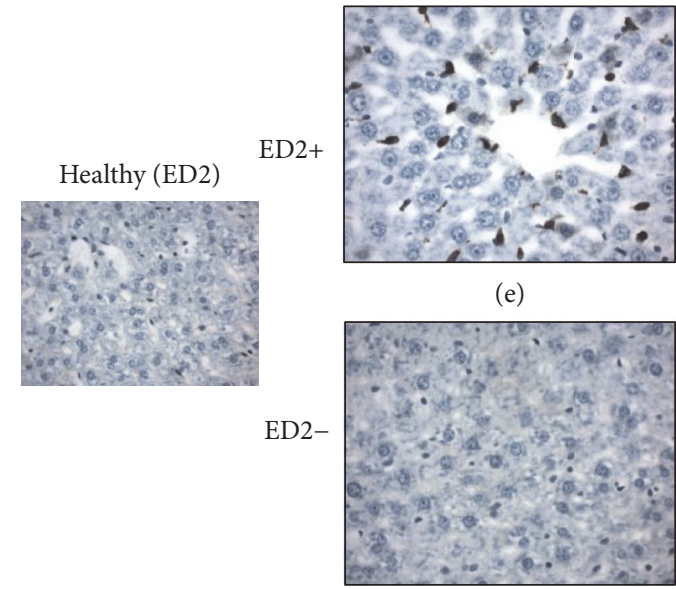

(g) 4x MTX

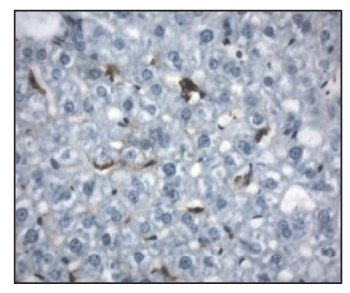

(b)

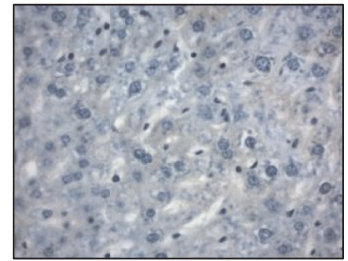

(d)

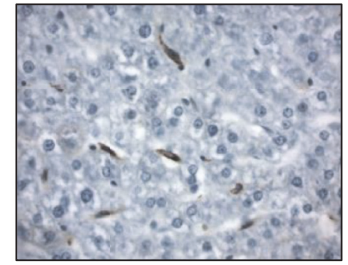

(f)

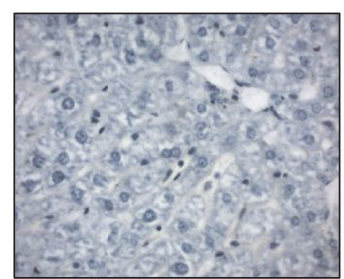

(h)

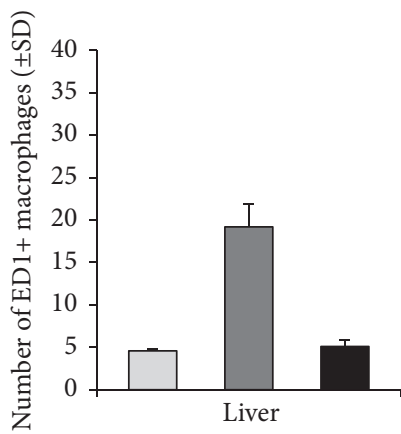

$\square$ Healthy

ㅁ Saline

- MTX

(i)

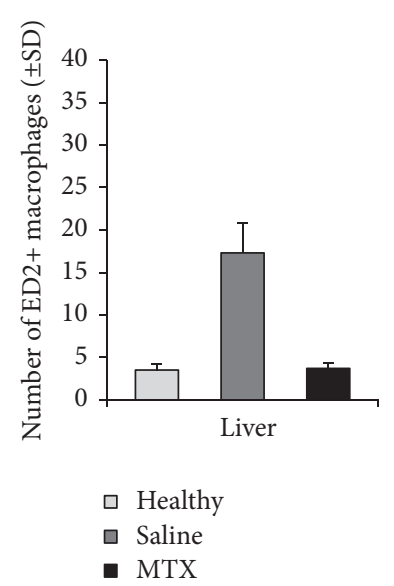

(j)

FIGURE 3: Representative immunohistochemical (HE) images of $\mathrm{ED}^{+}$and $\mathrm{ED} 2^{+}$macrophages in liver sections of healthy $(n=3)(\mathrm{ED} 1$ and ED2), saline-treated $(n=4)$, and MTX-treated $(n=4)$ rats. $((\mathrm{a}),(\mathrm{b}))$ Images represent $\mathrm{ED1}^{+}$macrophages in the liver of saline-treated and MTX-treated rats, respectively. ((c), (d)) Isotype control stained liver sections of saline-treated and MTX-treated rats, respectively. ((e), (f)) Images of ED2 ${ }^{+}$macrophages in the liver of saline-treated and MTX-treated rats, respectively. ((g), (h)) Images of isotype control stained liver sections of saline-treated and MTX-treated rats, respectively. ((i), (j)) Bar graph representations of quantifications of ED1 ${ }^{+}$and ED2 ${ }^{+}$ macrophages in liver of healthy, saline-treated, and MTX-treated rats. Values depict mean numbers of macrophages counted in predefined areas of the liver. Error bars indicate SD. $p<0.01$. 


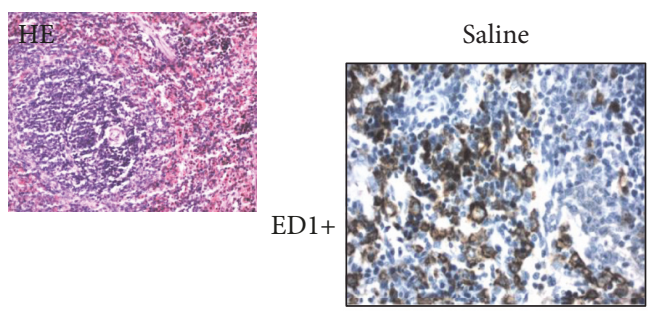

(a)
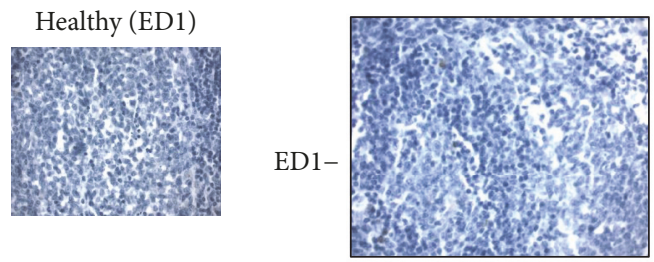

(c)

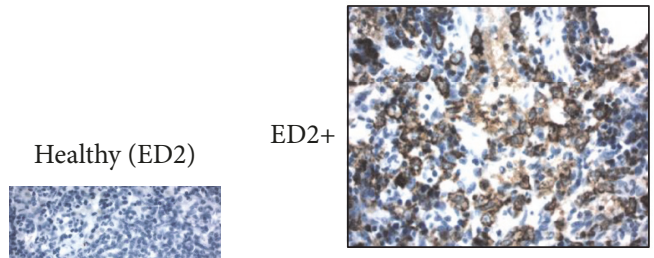

(e)

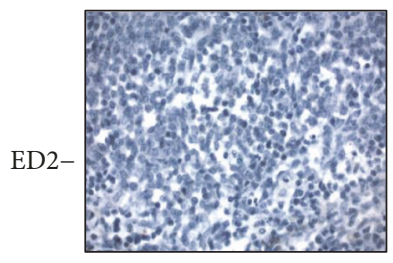

(g)

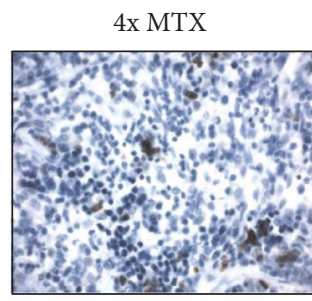

(b)

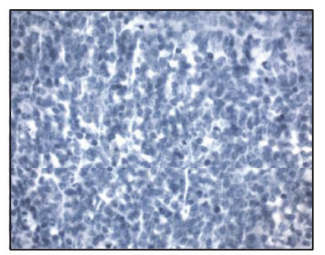

(d)

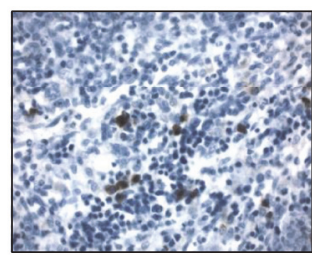

(f)

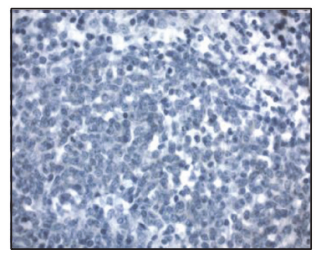

(h)

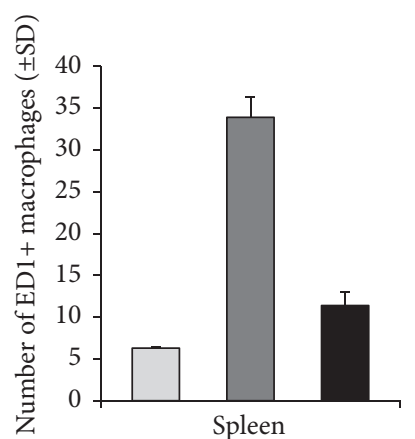

$\square$ Healthy

․ Saline

- MTX

(i)

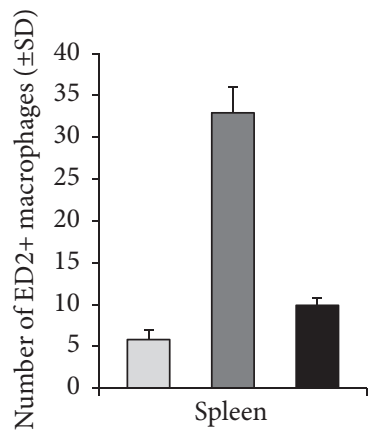

- Healthy

- MTX

(j)

FIGURE 4: Representative immunohistochemical (HE) images of ED1 ${ }^{+}$and ED2 $2^{+}$macrophages in spleen sections of healthy $(n=3)(\mathrm{ED} 1$ and ED2), saline-treated $(n=4)$, and MTX-treated $(n=4)$ rats. $((\mathrm{a}),(\mathrm{b}))$ Images represent $\mathrm{ED1}^{+}$macrophages in the spleen of saline-treated and MTX-treated rats, respectively. ((c), (d)) Isotype control stained spleen sections of saline-treated and MTX-treated rats, respectively. ((e), (f)) Images of ED2 ${ }^{+}$macrophages in the spleen of saline-treated and MTX-treated rats, respectively. $((\mathrm{g}),(\mathrm{h}))$ Images of isotype control stained spleen sections of saline-treated and MTX-treated rats, respectively. ((I), (J)) Bar graph representations of quantifications of ED1 ${ }^{+}$and ED2 ${ }^{+}$ macrophages in spleen of healthy, saline-treated, and MTX-treated rats. Values depict mean numbers of macrophages counted in predefined areas of the spleen. Error bars indicate SD. $p<0.01$.

in liver and spleen sections. In liver and spleen of arthritic rats the numbers of ED1- and ED2-positive macrophages were $\sim 5$ fold higher $(p<0.01)$ than those of healthy rats.

For both ED1- and ED2-positive macrophages in liver and spleen, a marked decrease in macrophage infiltration is noted for MTX treatment compared to saline-treated rats. This was confirmed by a significantly ( 4 -fold, $p<0.01$ ) lower numbers of ED1- and ED2-positive macrophages in the liver of MTXtreated rats (Figures $3(\mathrm{i})$ and $3(\mathrm{j})$ )), compared to saline-treated rats. Similarly, spleen sections of MTX-treated rats revealed significantly (3-fold, $p<0.01$ ) lower quantifications of ED1and ED2-positive macrophages, compared to saline-treated rats (Figures 4(i) and 4(j)). Antibody control stained liver and spleen sections were clearly negative for both ED1- and ED2-positive macrophages (Figures 3(c), 3(d), 3(g), 3(h), $4(\mathrm{c}), 4(\mathrm{~d}), 4(\mathrm{~g})$, and $4(\mathrm{~h}))$. It is of interest to note that MTX impacted the infiltration of both ED1 and ED2 macrophage in liver and spleen of arthritic rats. For ED2 macrophages this may be counterintuitive given their assigned antiinflammatory phenotype [13]. However, in the context of RA, recent evidence suggests that M2 macrophages can be skewed to produce proinflammatory cytokines [39], which can shift the balance of M2 to a more M1 phenotype. An alternative explanation could be that the MTX impacts circulating proinflammatory subsets of FR $\beta$ expressing circulating monocytes [40] to suppress overall infiltration and polarization of macrophages in arthritic knees, liver, and spleen. Unravelling the exact mechanism of action of how MTX impairs macrophage infiltration awaits further research.

3.5. Effect of MTX on FR $\beta$-Positive Macrophages. FR $\beta$ positive synovial macrophages were shown to be highly infiltrated in the synovium of RA patients [27]. Given that $\left[{ }^{18} \mathrm{~F}\right]$ fluoro-PEG-folate binds to FR $\beta$ [25], we examined the 


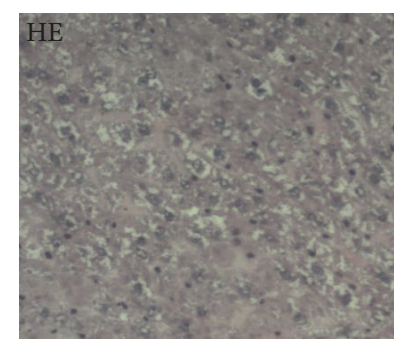

Saline

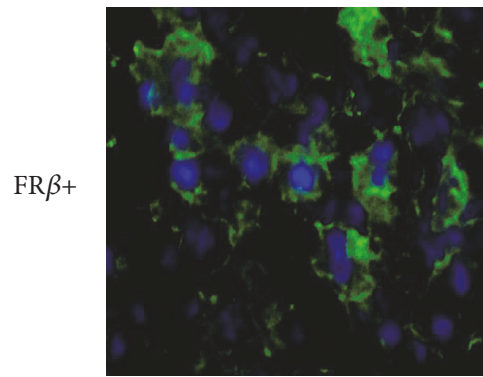

(a)

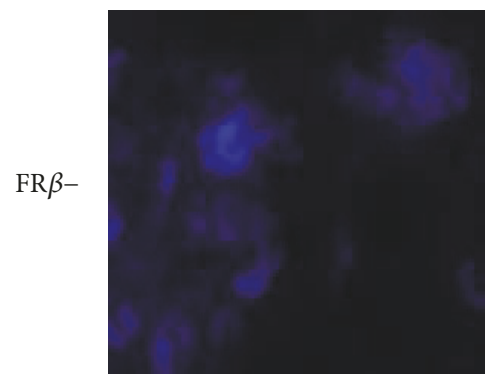

(c)

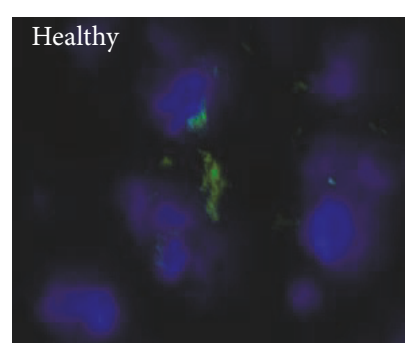

4x MTX

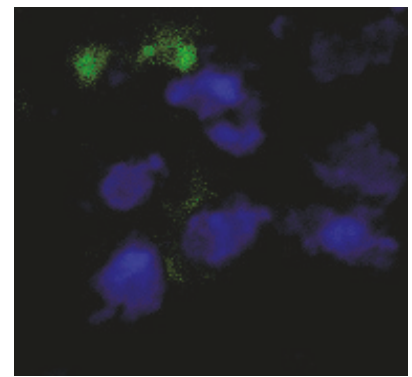

(b)

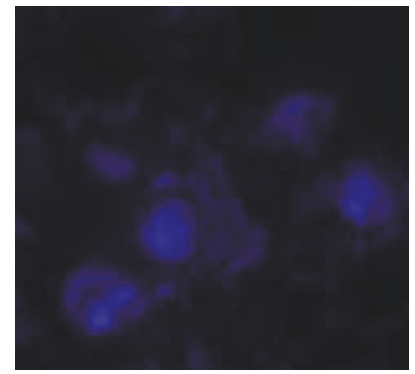

(d)

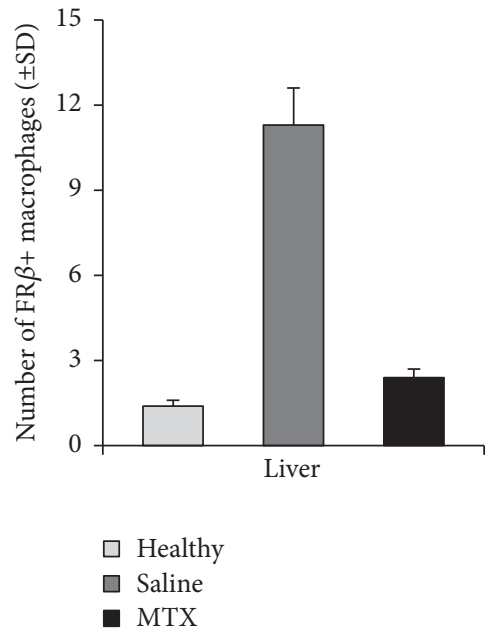

(e)

FIGURE 5: Representative immunofluorescence images of FR $\beta^{+}$macrophages in liver sections of healthy $(n=3)$ and saline- $(n=4)$ and MTXtreated $(n=4)$ rats. ( (a), (b)) Images represent FR $\beta^{+}$macrophages in the liver of saline-treated and MTX-treated rats, respectively. ((c), (d)) Isotype control stained liver sections of saline-treated and MTX-treated rats, respectively. (e) Bar graph representation of quantifications of $\mathrm{FR} \beta^{+}$macrophages in liver of saline-treated and MTX-treated rats. Values depict mean numbers of macrophages counted in predefined areas of the liver. Error bars indicate SD (blue color: DAPI (nucleus staining); green color: FR $\beta$ staining). $p<0.01$.

expression of FR $\beta$ in liver and spleen sections of salinetreated and MTX-treated arthritic rats to verify the data of the PET and tissue distribution studies. In liver and spleen of arthritic rats the number of $\mathrm{FR} \beta$-positive macrophages was significantly $(p<0.01)$ higher than those of healthy rats.

Representative immunofluorescence images of $\operatorname{FR} \beta$ expression in cryosections of liver (Figures 5(a)-5(d)) and spleen (Figures 6(a)-6(d)) after saline and MTX therapeutic interventions revealed a markedly lower FR $\beta$ expression in both liver and spleen of MTX-treated versus salinetreated rats. This was confirmed by quantitative assessments showing significant 5-fold $(p<0.005)$ and 3-fold $(p<0.01)$ lower numbers of FR $\beta$-positive macrophages in the liver (Figure 5(e)) and spleen (Figure 6(e)) of MTX-treated rats. The FR $\beta$ levels in MTX-treated rats approximated FR $\beta$ positive macrophages in liver and spleen of healthy rats. Antibody control stained liver and spleen sections were negative (Figures 5(c), 5(d), 6(c), and 6(d)). Results for FR $\beta$ staining were consistent with ED1 and ED2 stainings (Figures 3 and 4). Together, these results underscore that macrophage infiltration in liver and spleen is implicated in inflammation and response to therapy, similar to that shown for RA synovium in patients [14-16].

In addition to MTX, antiarthritic effects elicited through FR $\beta$ targeting have been reported for folate-conjugated immunotoxins [29] and various folate-conjugated drugs $[30,41,42]$. Since FR $\beta$ is primarily expressed on activated macrophages $[27,28]$, microenvironmental conditions in liver and spleen will be of importance for FR $\beta$ expression and macrophage polarization. FR $\beta$ expression has been reported on both M1- and M2-type macrophages [43], and in rat RA synovium FR $\beta$ expression has been also observed on a mixed M1- and M2-type [44]. As indicated above, in the RA microenvironment with circulating complex IgG autoantibodies and/or ACPA antibodies, FR $\beta$ expressing activated macrophages can release proinflammatory cytokines [39, 45] and thus be a bona fide target. More detailed investigations on the specific polarization and phenotypic properties of FR $\beta$ expressing tissue macrophages in liver and spleen may assist optimal targeting of this receptor for imaging and therapeutic 


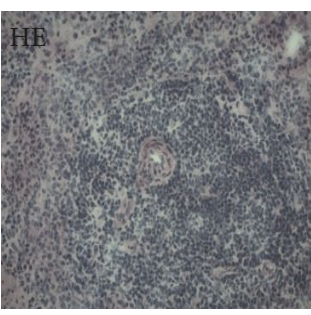

Saline

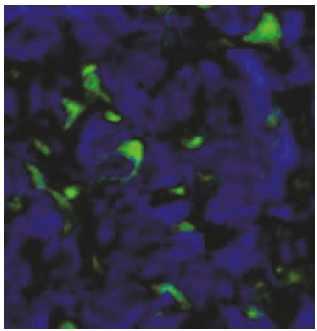

(a)

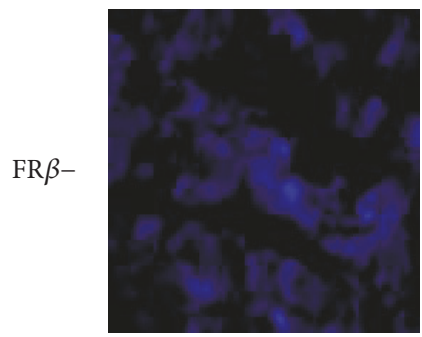

(c)

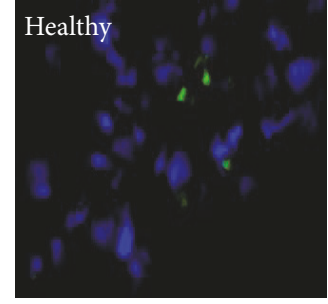

4x MTX

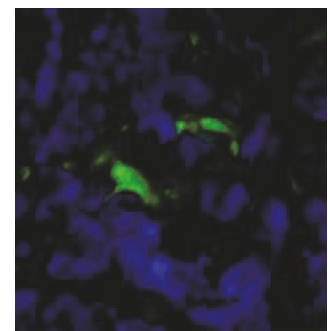

(b)

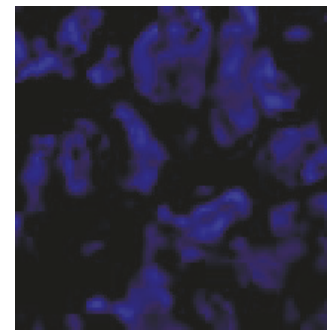

(d)

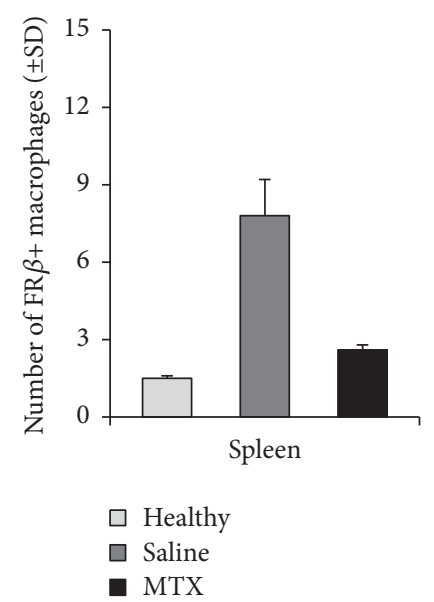

(e)

FIGURE 6: Representative immunofluorescence images of FR $\beta^{+}$macrophages in spleen sections of healthy $(n=3)$ and saline- $(n=4)$ and MTX-treated $(n=4)$ rats. ((a), (b)) Images represent FR $\beta^{+}$macrophages in the spleen of saline-treated and MTX-treated rats, respectively. ((c), (d)) Isotype control stained spleen sections of saline-treated and MTX-treated rats, respectively. (e) Bar graph representation of quantifications of $\mathrm{FR} \beta^{+}$macrophages in spleen of saline-treated and MTX-treated rats. Values depict mean numbers of macrophages counted in predefined areas of the spleen. Error bars indicate SD. (blue color: DAPI (nuclear staining); green color: FR $\beta$ staining). $p<0.01$.

exploitations. These premises do not only hold for arthritis but also for cancer [46].

\section{Conclusion}

MTX treatment reduced activated macrophages in liver and spleen, as markers for systemic inflammation in these organs. Macrophage PET imaging with $\left[{ }^{18} \mathrm{~F}\right]$ fluoro-PEG-folate holds promise for detection of systemic inflammation in RA as well as therapy (MTX) response monitoring.
Abbreviations
RA: Rheumatoid arthritis
mBSA: Methylated bovine serum albumin
MTX: Methotrexate
HRRT: High resolution research tomography
PEG: Polyethylene glycol
SD: $\quad$ Standard deviation
$\%$ ID/g: Percentage of the injected dose per gram
i.a.: Intra-articular
FR $\beta$ : Folate Receptor $\beta$

ROI: Region of interest

EC20: A folate-linked chelator of 99mTc.

\section{Conflicts of Interest}

The authors declare that they have no conflicts of interest regarding the publication of this article.

\section{Authors' Contributions}

Durga M. S. H. Chandrupatla contributed to the acquisition, analysis, and interpretation of the data and drafted the manuscript. Elise Mantel was involved in all in vitro studies. Carla F. M. Molthoff, Gerrit Jansen, and Conny J. van der Laken were involved in the design of the study, contributed to the interpretation of the data, and critically revised the manuscript. Adriaan A. Lammertsma contributed to the interpretation of the PET data and critically revised the manuscript. Philip S. Low synthesized the PET tracer precursor and assisted with revision of the manuscript. Albert D. Windhorst synthesized the folate PET tracer and performed quality controls on the tracer. Takami Matsuyama 
provided the mouse anti-rat-folate receptor $\beta$ antibody and staining protocol and critically revised the manuscript. René P. Musters was involved in the acquisition and interpretation of the immunofluorescence data and critically revised the manuscript. All authors read and approved the final manuscript.

\section{Acknowledgments}

The authors gratefully acknowledge Mariska Verlaan and Ricardo Vos for their excellent technical support with the PET scans and animal experiments. Rolph van Kooij and Martien Mooijer are thanked for $\left[{ }^{18} \mathrm{~F}\right]$ fluoro-PEG-folate tracer synthesis and planning. This study was supported by VU University Medical Center (Cancer Center Amsterdam) (CCA-PV13/87).

\section{References}

[1] J. S. Smolen, D. Aletaha, and I. B. McInnes, "Rheumatoid arthritis," The Lancet, vol. 388, no. 10055, pp. 2023-2038, 2016.

[2] E. Choy, "Understanding the dynamics: Pathways involved in the pathogenesis of rheumatoid arthritis," Rheumatology, vol. 51, supplement 5, pp. v3-v11, 2012.

[3] M. Cojocaru, I. M. Cojocaru, I. Silosi, C. D. Vrabie, and R. Tanasescu, "Extra-articular Manifestations in Rheumatoid Arthritis," Maedica, vol. 5, no. 4, pp. 286-291, 2010.

[4] I. B. McInnes and G. Schett, "The pathogenesis of rheumatoid arthritis," The New England Journal of Medicine, vol. 365, no. 23, pp. 2205-2219, 2011.

[5] Y. P. M. Goekoop-Ruiterman, J. K. de Vries-Bouwstra, C. F. Allaart et al., "Clinical and radiographic outcomes of four different treatment strategies in patients with early rheumatoid arthritis (the best study): a randomized, controlled trial," Arthritis \& Rheumatology, vol. 52, no. 11, pp. 3381-3390, 2005.

[6] M. F. Bakker, J. W. G. Jacobs, S. M. M. Verstappen, and J. W. J. Bijlsma, "Tight control in the treatment of rheumatoid arthritis: efficacy and feasibility," Annals of the Rheumatic Diseases, vol. 66, no. 3, pp. iii56-iii60, 2007.

[7] G. Kollias, P. Papadaki, F. Apparailly et al., "Animal models for arthritis: Innovative tools for prevention and treatment," Annals of the Rheumatic Diseases, vol. 70, no. 8, pp. 1357-1362, 2011.

[8] P. Li and E. M. Schwarz, "The TNF- $\alpha$ transgenic mouse model of inflammatory arthritis," Springer Seminars in Immunopathology, vol. 25, no. 1, pp. 19-33, 2003.

[9] A. D. Christensen, C. Haase, A. D. Cook, and J. A. Hamilton, "K/BxN serum-transfer arthritis as a model for human inflammatory arthritis," Frontiers in Immunology, vol. 7, article no. 213, 2016.

[10] R. C. Schimmer, D. J. Schrier, C. M. Flory et al., "Streptococcal cell wall-induced arthritis: requirements for IL-4, IL- 10, IFN$\gamma$, and monocyte chemoattractant protein-1," The Journal of Immunology, vol. 160, no. 3, pp. 1466-1471, 1998.

[11] L. Bevaart, M. J. Vervoordeldonk, and P. P. Tak, "Evaluation of therapeutic targets in animal models of arthritis: how does it relate to rheumatoid arthritis?" Arthritis \& Rheumatology, vol. 62, no. 8, pp. 2192-2205, 2010.

[12] B. D. Fischer, A. Adeyemo, M. E. O'Leary, and A. Bottaro, "Animal models of rheumatoid pain: Experimental systems and insights," Arthritis Research \& Therapy, vol. 19, no. 1, article no. 146, 2017.
[13] I. A. Udalova, A. Mantovani, and M. Feldmann, "Macrophage heterogeneity in the context of rheumatoid arthritis," Nature Reviews Rheumatology, vol. 12, no. 8, pp. 472-485, 2016.

[14] Z. N. Jahangier, J. W. G. Jacobs, M. C. Kraan et al., "Pretreatment macrophage infiltration of the synovium predicts the clinical effect of both radiation synovectomy and intra-articular glucocorticoids," Annals of the Rheumatic Diseases, vol. 65, no. 10, pp. 1286-1292, 2006.

[15] M. D. Smith, M. C. Kraan, J. Slavotinek et al., "Treatmentinduced remission in rheumatoid arthritis patients is characterized by a reduction in macrophage content of synovial biopsies," Rheumatology, vol. 40, no. 4, pp. 367-374, 2001.

[16] J. J. Haringman, D. M. Gerlag, A. H. Zwinderman et al., "Synovial tissue macrophages: a sensitive biomarker for response to treatment in patients with rheumatoid arthritis," Annals of the Rheumatic Diseases, vol. 64, no. 6, pp. 834-838, 2005.

[17] L. C. Davies, S. J. Jenkins, J. E. Allen, and P. R. Taylor, "Tissueresident macrophages," Nature Immunology, vol. 14, no. 10, pp. 986-995, 2013.

[18] C. Selmi, M. De Santis, and M. E. Gershwin, "Liver involvement in subjects with rheumatic disease," Arthritis Research \& Ther$a p y$, vol. 13, no. 3, article 226, 2011.

[19] Y. Kakinuma, T. Kimura, and Y. Watanabe, "Possible Involvement of Liver Resident Macrophages (Kupffer Cells) in the Pathogenesis of Both Intrahepatic and Extrahepatic Inflammation," Canadian Journal of Gastroenterology and Hepatology, vol. 2017, Article ID 2896809, 10 pages, 2017.

[20] K. Nishiya, N. Hisakawa, T. Hosokawa, K. Hashimoto, and T. Doi, "Enlarged spleen detected by abdominal ultrasonography in patients with RA," Annals of the Rheumatic Diseases, vol. 59, no. 9, pp. 751-752, 2000.

[21] J. M. Pena, J. Garcia-Alegria, M. Crespo, J. Gijon, and J. J. Vazquez, "Spontaneous rupture of the spleen in RA," Annals of the Rheumatic Diseases, vol. 43, no. 3, pp. 411-414, 1984.

[22] S. T. G. Bruijnen, Y. Y. J. Gent, A. E. Voskuyl, O. S. Hoekstra, and C. J. Van Der Laken, "Present role of positron emission tomography in the diagnosis and monitoring of peripheral inflammatory arthritis: A systematic review," Arthritis Care \& Research, vol. 66, no. 1, pp. 120-130, 2014.

[23] Y. Y. J. Gent, K. Weijers, C. F. M. Molthoff et al., "Promising potential of new generation translocator protein tracers providing enhanced contrast of arthritis imaging by positron emission tomography in a rat model of arthritis," Arthritis Research \& Therapy, vol. 16, no. 2, article R70, 2014.

[24] S. A. Kularatne, M.-J. Bélanger, X. Meng et al., "Comparative analysis of folate derived PET imaging agents with [18F]-2fluoro-2-deoxy-d-glucose using a rodent inflammatory paw model," Molecular Pharmaceutics, vol. 10, no. 8, pp. 3103-3111, 2013.

[25] Y. Y. J. Gent, K. Weijers, C. F. M. Molthoff et al., "Evaluation of the novel folate receptor ligand [18F]fluoro-PEG-folate for macrophage targeting in a rat model of arthritis," Arthritis Research \& Therapy, vol. 15, no. 2, article R37, 2013.

[26] N. Nakashima-Matsushita, T. Homma, S. Yu et al., "Selective expression of folate receptor $\beta$ and its possible role in methotrexate transport in synovial macrophages from patients with rheumatoid arthritis," Arthritis \& Rheumatology, vol. 42, no. 8, pp. 1609-1616, 1999.

[27] J. W. van der Heijden, R. Oerlemans, B. A. C. Dijkmans et al., "“Folate receptor beta as a potential delivery route for novel folate antagonists to macrophages in the synovial tissue of 
rheumatoid arthritis patients," Arthritis \& Rheumatology, vol. 60, no. 1, pp. 12-21, 2009.

[28] W. Xia, A. R. Hilgenbrink, E. L. Matteson, M. B. Lockwood, J.X. Cheng, and P. S. Low, "A functional folate receptor is induced during macrophage activation and can be used to target drugs to activated macrophages," Blood, vol. 113, no. 2, pp. 438-446, 2009.

[29] T. Nagai, A. Kyo, K. Hasui, S. Takao, and T. Matsuyama, "Efficacy of an immunotoxin to folate receptor beta in the intra-articular treatment of antigen-induced arthritis," Arthritis Research \& Therapy, vol. 14, no. 3, article R106, 2012.

[30] Y. Lu, N. Parker, P. J. Kleindl et al., "Antiinflammatory activity of a novel folic acid targeted conjugate of the mTOR inhibitor everolimus," Molecular Medicine, vol. 21, pp. 584-596, 2015.

[31] R. Nagayoshi, M. Nakamura, K. Ijiri, H. Yoshida, S. Komiya, and T. Matsuyama, "LY309887, antifolate via the folate receptor suppresses murine type II collagen-induced arthritis," Clinical and Experimental Rheumatology, vol. 21, no. 6, pp. 719-725, 2003.

[32] D. M. S. H. Chandrupatla, G. Jansen, R. Vos et al., "Invivo monitoring of anti-folate therapy in arthritic rats using [18F]fluoro-PEG-folate and positron emission tomography," Arthritis Research \& Therapy, vol. 19, no. 1, article no. 114, 2017.

[33] D. M. S. H. Chandrupatla, K. Weijers, Y. Y. J. Gent et al., "Sustained macrophage infiltration upon multiple intra-articular injections: An improved rat model of rheumatoid arthritis for PET guided therapy evaluation," BioMed Research International, vol. 2015, Article ID 509295, 11 pages, 2015.

[34] A. M. Loening and S. S. Gambhir, "AMIDE: a free software tool for multimodality medical image analysis," Molecular Imaging, vol. 2, no. 3, pp. 131-137, 2003.

[35] S. E. Verbrugge, M. Al, Y. G. Assaraf et al., "Multifactorial resistance to aminopeptidase inhibitor prodrug CHR2863 in myeloid leukemia cells: Down-regulation of carboxylesterase 1, drug sequestration in lipid droplets and pro-survival activation ERK/Akt/mTOR," Oncotarget, vol. 7, no. 5, pp. 5240-5257, 2016.

[36] M. J. Turk, G. J. Breur, W. R. Widmer et al., "Folate-targeted imaging of activated macrophages in rats with adjuvantinduced arthritis," Arthritis \& Rheumatology, vol. 46, no. 7, pp. 1947-1955, 2002.

[37] T. Okabe, H. Shibata, K. Shizukuishi, T. Yoneyama, T. Inoue, and U. Tateishi, "F-18 FDG uptake patterns and disease activity of collagen vascular diseases-associated arthritis," Clinical Nuclear Medicine, vol. 36, no. 5, pp. 350-354, 2011.

[38] E. L. Matteson, V. J. Lowe, F. G. Prendergast et al., "Assessment of disease activity in rheumatoid arthritis using a novel folate targeted radiopharmaceutical Folatescan," Clinical and Experimental Rheumatology, vol. 27, no. 2, pp. 253-259, 2009.

[39] C. Clavel, L. Ceccato, F. Anquetil, G. Serre, and M. Sebbag, "Among human macrophages polarised to different phenotypes, the M-CSF-oriented cells present the highest pro-inflammatory response to the rheumatoid arthritis-specific immune complexes containing ACPA," Annals of the Rheumatic Diseases, vol. 75, no. 12, pp. 2184-2191, 2016.

[40] J. Shen, A. R. Hilgenbrink, W. Xia et al., "Folate receptor- $\beta$ constitutes a marker for human proinflammatory monocytes," Journal of Leukocyte Biology, vol. 96, no. 4, pp. 563-570, 2014.

[41] Y. Lu, T. W. Stinnette, E. Westrick et al., "Treatment of experimental adjuvant arthritis with a novel folate receptor-targeted folic acid-aminopterin conjugate," Arthritis Research \& Therapy, vol. 13, no. 2, article no. R56, 2011.
[42] Y. Yi, "Folate receptor-targeted diagnostics and therapeutics for inflammatory diseases," Immune Network, vol. 16, no. 6, pp. 337343, 2016.

[43] A. Puig-Kröger, E. Sierra-Filardi, A. Domínguez-Soto et al., "Folate receptor $\beta$ is expressed by tumor-associated macrophages and constitutes a marker for M2 anti-inflammatory/ regulatory Macrophages," Cancer Research, vol. 69, no. 24, pp. 9395-9403, 2009.

[44] Y. Tsuneyoshi, M. Tanaka, T. Nagai et al., "Functional folate receptor beta-expressing macrophages in osteoarthritis synovium and their M1/M2 expression profiles," Scandinavian Journal of Rheumatology, vol. 41, no. 2, pp. 132-140, 2012.

[45] L. T. C. Vogelpoel, I. S. Hansen, T. Rispens et al., "Fc gamma receptor-TLR cross-talk elicits pro-inflammatory cytokine production by human M2 macrophages," Nature Communications, vol. 5, article no. 5444, 2014.

[46] J. Shen, K. S. Putt, D. W. Visscher et al., "Assessment of folate receptor- $\beta$ expression in human neoplastic tissues," Oncotarget , vol. 6, no. 16, pp. 14700-14709, 2015. 


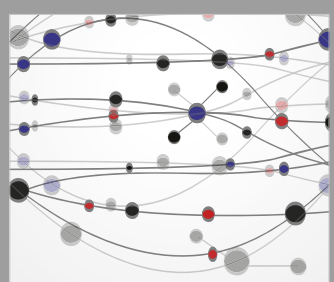

The Scientific World Journal
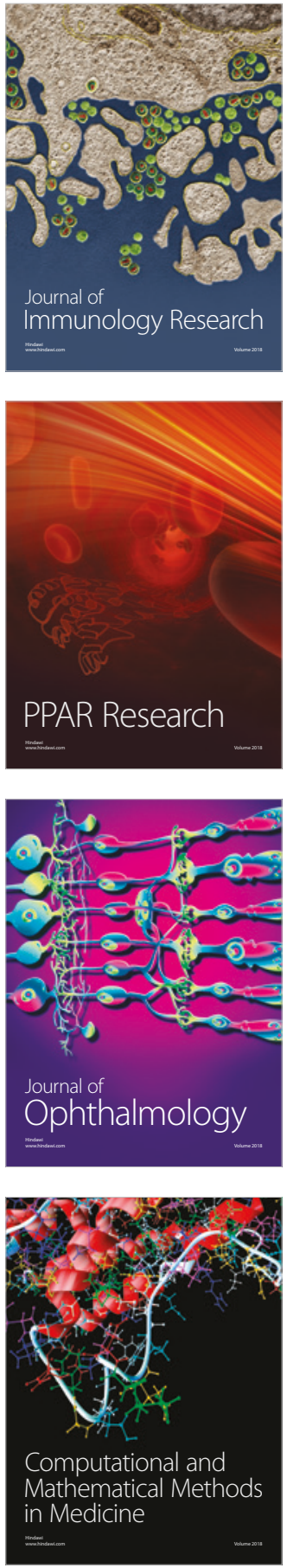

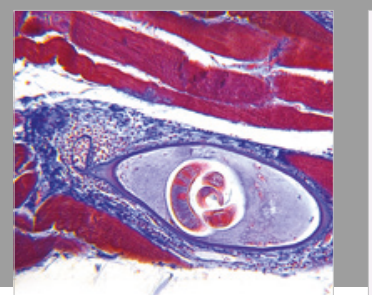

Gastroenterology Research and Practice

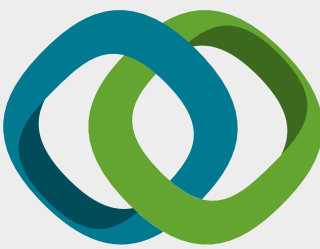

\section{Hindawi}

Submit your manuscripts at

www.hindawi.com
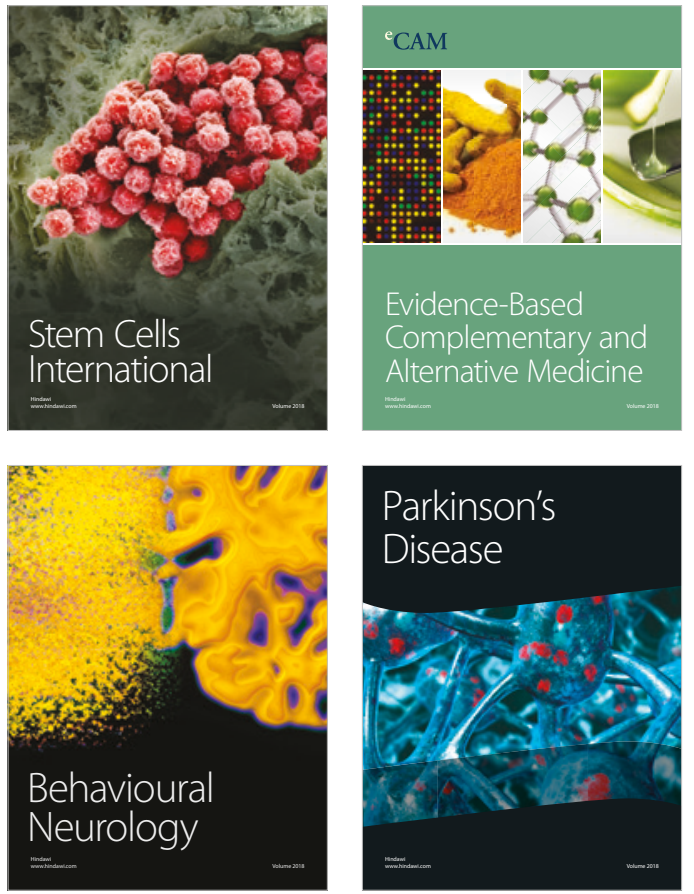

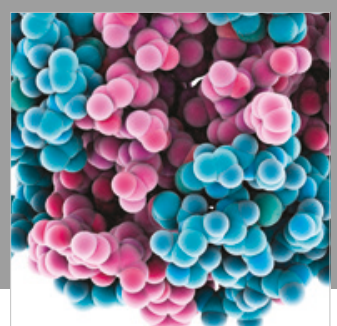

ournal of

Diabetes Research

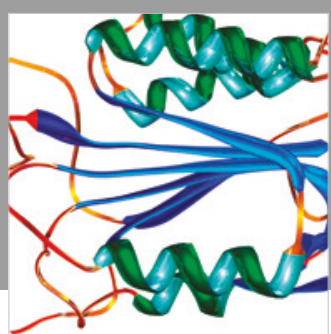

Disease Markers
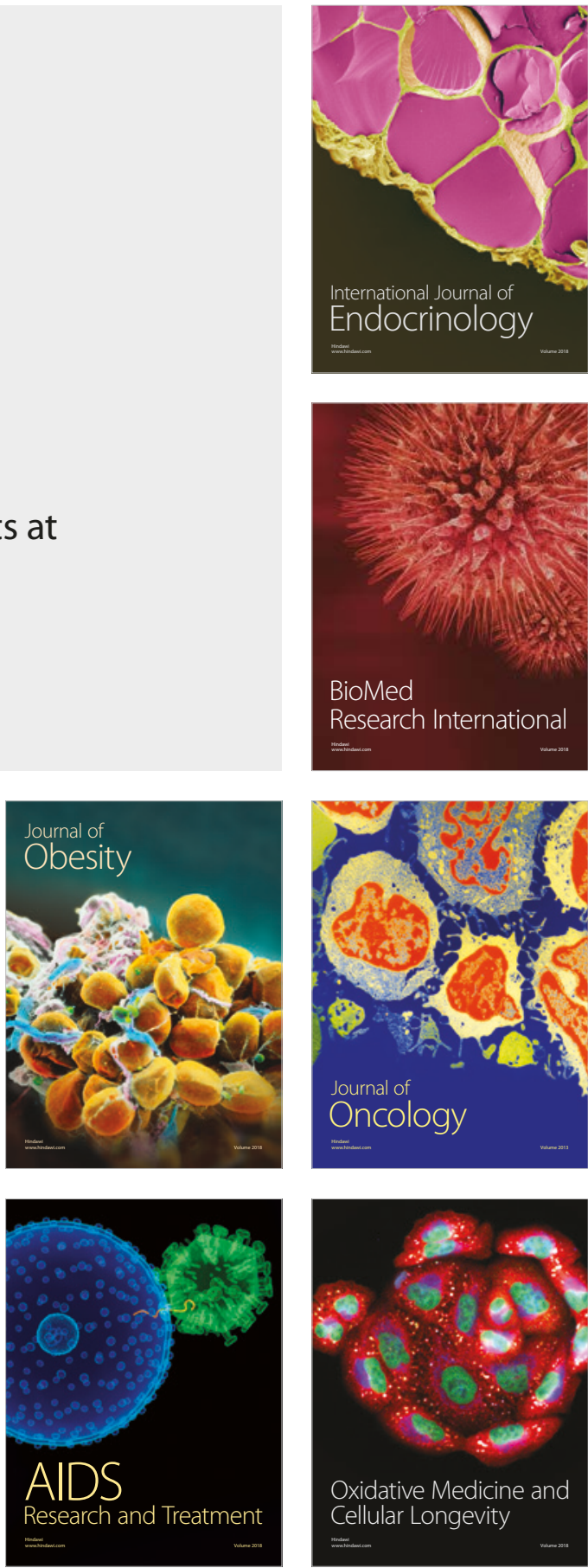